\title{
The Isomorphism Problem for $k$-Trees is Complete for Logspace
}

\author{
Johannes Köbler and Sebastian Kuhnert \\ Institut für Informatik, Humboldt Universität zu Berlin, Germany, \\ \{koebler, kuhnert\}@informatik.hu-berlin.de
}

\begin{abstract}
We show that $k$-tree isomorphism can be decided in logarithmic space by giving a logspace canonical labeling algorithm. This improves over the previous StUL upper bound and matches the lower bound. As a consequence, the isomorphism, the automorphism, as well as the canonization problem for $k$-trees are all complete for deterministic logspace. We also show that even simple structural properties of $k$-trees are complete for logspace.

Keywords: graph isomorphism, graph canonization, k-trees, space complexity, logspace completeness.
\end{abstract}

\section{Introduction}

Two graphs $G$ and $H$ are called isomorphic if there is a bijective mapping $\phi$ between the vertices of $G$ and the vertices of $H$ that preserves the adjacency relation, i.e., $\phi$ relates edges to edges and non-edges to non-edges. Graph Isomorphism (GI) is the problem of deciding whether two given graphs are isomorphic. The problem has received considerable attention since it is one of the few natural problems in NP that are neither known to be NP-complete nor known to be solvable in polynomial time.

It is known that GI is contained in coAM [GS86,Sch88] and in SPP [AK06] providing strong evidence that GI is not NP-complete. On the other hand, the strongest known hardness result due to Torán [Tor04] says that GI is hard for the class DET (cf. [Coo85]). DET is a subclass of $\mathrm{NC}^{2}$ (even of $\mathrm{TC}^{1}$ ) and contains $\mathrm{NL}$ as well as all logspace counting classes [AJ93, $\mathrm{BDH}^{+}$92].

For some restricted graph classes the known upper and lower complexity bounds for the isomorphism problem match. For example, a linear time algorithm for tree isomorphism was already known in 1974 to Aho, Hopcroft and Ullman [AHU74]. In 1991, an NC algorithm was developed by Miller and Reif [MR91], and one year later, Lindell [Lin92] obtained an L upper bound. On the other hand, in $\left[\mathrm{JKM}^{+} 03\right]$ it is shown that tree isomorphism is L-hard (provided that the trees are given in pointer notation). In [ADK08], Lindell's log-space upper bound has been extended to the class of partial 2-trees, a class of planar graphs also known as generalized series-parallel graphs. Very recently, it has been shown that even the isomorphism problem for all planar graphs is in logspace $\left[\mathrm{DLN}^{+} 08\right]$. Much of the recent progress on logspace algorithms for 
graphs has only become possible through Reingold's result that connectivity in undirected graphs can be decided in deterministic logspace [Rei05]. Our result does not depend on this, yielding a comparatively simple algorithm.

In this paper we show that the isomorphism problem for $k$-trees is in logspace for each fixed $k \in \mathbb{N}^{+}$. This improves the previously known upper bound of StUL [ADK07] and matches the lower bound. In fact, we prove the formally stronger result that a canonical labeling for a given $k$-tree is computable in logspace. Recall that the canonization problem for graphs is to produce a canonical form $\operatorname{canon}(G)$ for a given graph $G$ such that $\operatorname{canon}(G)$ is isomorphic to $G$ and $\operatorname{canon}\left(G_{1}\right)=\operatorname{canon}\left(G_{2}\right)$ for any pair of isomorphic graphs $G_{1}$ and $G_{2}$. Clearly, graph isomorphism reduces to graph canonization. A canonical labeling for $G$ is any isomorphism between $G$ and $\operatorname{canon}(G)$. It is not hard to see that even the search version of GI (i.e., computing an isomorphism between two given graphs in case it exists) as well as the automorphism group problem (i.e., computing a generating set of the automorphism group of a given graph) are both logspace reducible to the canonical labeling problem.

The parallel complexity of $k$-tree isomorphism has been previously investigated by Del Greco, Sekharan, and Sridhar [GSS02] who introduced the concept of the kernel of a $k$-tree in order to restrict the search for an isomorphism between two given $k$-trees. We show that the kernel of a $k$-tree can be computed in logspace and exploit this fact to restrict the search for a canonical labeling of a given $k$-tree $G$. To be more precise, we first transform $G$ into an undirected tree $T(G)$ whose nodes are formed by the $k$-cliques and $(k+1)$-cliques of $G$. Then we compute the center node of $T(G)$ which coincides with the kernel of $G$ and try all labelings of the vertices in $\operatorname{ker}(G)$. In order to extend a labeling of the kernel vertices of $G$ to the other vertices of $G$ in a canonical way, we color the nodes of the tree $T(G)$ to encode additional structural information about $G$. Finally, we apply a variant of Lindell's algorithm to compute canonical labelings for the colored versions of $T(G)$ and derive from them a canonical labeling for the $k$-tree $G$.

Our tree representation $T(G)$ is similar to the construction used in [ADK07]. The main advantage of our construction lies in the fact that the tree $T(G)$ can be directly constructed in logspace from $G$, whereas the tree representation of [ADK07] is obtained as a reachable subgraph of a mangrove ${ }^{1}$ based on $G$ and hence can only be derived from $G$ with the help of an StUL oracle.

\section{Preliminaries}

As usual, $\mathbf{L}$ is the class of all languages decidable by Turing machines with readonly input tape and an $\mathcal{O}(\log n)$ bound on the space used on the working tapes. $\mathbf{F L}$ is the class of all functions computable by Turing machines that additionally have a write-only output tape.

Given a graph $G$, we use $\boldsymbol{V}(\boldsymbol{G})$ and $\boldsymbol{E}(\boldsymbol{G})$ to denote its vertex and edge sets, respectively. We define the following notations for subgraphs of $G$. For

\footnotetext{
${ }^{1}$ A mangrove is a digraph with at most one directed path between each pair of nodes.
} 
$M \subseteq V(G), \boldsymbol{G}[\boldsymbol{M}]$ denotes the subgraph of $G$ induced by $M$ and we use $\boldsymbol{G}-\boldsymbol{M}$ as a shorthand for $G[V(G) \backslash M]$.

Given a graph $G$ and two vertices $u, v \in V(G)$, the distance $\boldsymbol{d}_{G}(\boldsymbol{u}, \boldsymbol{v})$ is the length of the shortest path from $u$ to $v$. The eccentricity of a vertex $v \in V(G)$ is the longest distance to another vertex, i.e., $\boldsymbol{e c c}_{G}(\boldsymbol{v})=\max \left\{d_{G}(u, v) \mid u \in V(G)\right\}$. The center of $G$ consists of all vertices with minimal eccentricity.

Given two graphs $G$ and $H$, an isomorphism from $G$ to $H$ is a bijection $\phi: V(G) \rightarrow V(H)$ with $\{u, v\} \in E(G) \Leftrightarrow\{\phi(u), \phi(v)\} \in E(H)$. On colored graphs, an isomorphism must additionally preserve colors. $G$ and $H$ are called isomorphic, in symbols $G \cong H$, if there is an isomorphism from $G$ to $H$. Given a graph class $\mathcal{G}$, a function $f$ defined on $\mathcal{G}$ computes an invariant for $\mathcal{G}$ if

$$
\forall G, H \in \mathcal{G}: G \cong H \Rightarrow f(G)=f(H) .
$$

If the reverse implication also holds, $f$ is a complete invariant for $\mathcal{G}$. If additionally $f(G) \cong G$ for all $G \in \mathcal{G}, f$ computes canonical forms for $\mathcal{G}$. Given a function $f$ that computes canonical forms, an isomorphism $\psi_{G}$ from $G$ to its canonical form $f(G)$ is called a canonical labeling.

The isomorphisms from a graph $G$ to itself are called automorphisms and they form a group, which we denote by $\operatorname{Aut}(\boldsymbol{G})$. An automorphism is called non-trivial if it is not the identity. The graph automorphism problem (GA) is to decide if a graph has non-trivial automorphisms. A graph without non-trivial automorphisms is called rigid.

In the next section, we present an $\mathrm{FL}$ algorithm that, given a $k$-tree $G$, computes a canonical labeling $\psi_{G}$.

\section{Canonizing $k$-trees}

Fix any $k \in \mathbb{N}^{+}$. The class of $\boldsymbol{k}$-trees is inductively defined as follows. Any $k$-clique is a $k$-tree. Further, given a $k$-tree $G$ and a $k$-clique $C$ in $G$, one can construct another $k$-tree by adding a new vertex $v$ and connecting $v$ to every vertex in $C$. The initial $k$-clique is called base of $G$, and the $k$-clique $C$ the new vertex $v$ is connected to is called support of $v$. Note that each $k$-clique of a $k$-tree $G$ can be used as base for constructing $G$-but once the base is fixed, the support of each vertex is uniquely determined.

An interesting special case of $k$-trees are $\boldsymbol{k}$-paths, where the support $C_{i}$ of any new vertex $v_{i}$ (except the first vertex added to $G$ ) must either contain the vertex $v_{i-1}$ added in the previous step or be equal to the support $C_{i-1}$ of the latter. Fig. 1 shows a 2-tree that is a 2-path as well.

We note that $k$-trees can be recognized in logspace [ADK07], so we can safely assume that the input is indeed a $k$-tree.

We first define a tree representation $T(G)$ for $k$-trees $G$.

Definition 1. For a $k$-tree $G$, its tree representation $\boldsymbol{T}(G)$ is defined by

$$
\begin{aligned}
& V(T(G))=\{M \subseteq V(G) \mid M \text { is a } k \text {-clique or a }(k+1) \text {-clique }\} \\
& E(T(G))=\left\{\left\{M_{1}, M_{2}\right\} \subseteq V(T(G)) \mid M_{1} \subsetneq M_{2}\right\} .
\end{aligned}
$$



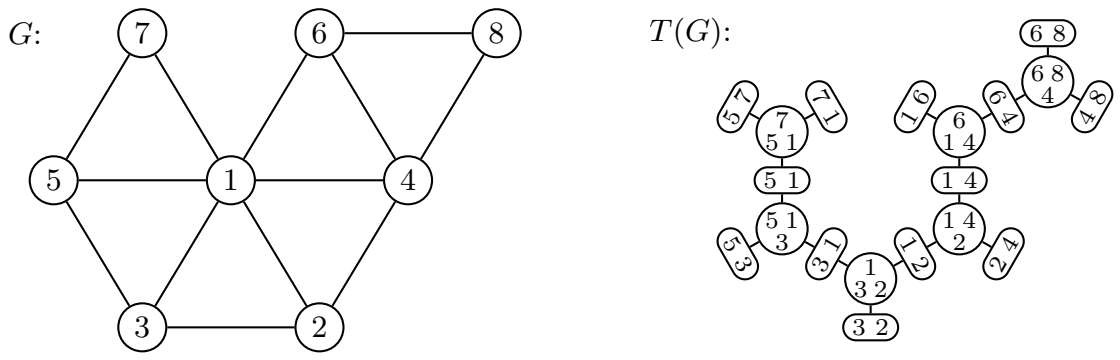

Fig. 1. A 2-tree $G$ and its tree representation $T(G)$

Note that $T(G)$ reflects the iterative construction of $G$ : The base of $G$ is a $k$-clique and thus a node in $T(G)$. Each time a new vertex $u$ is added to $G$, it is connected to all vertices of its support $P_{u}$ (a $k$-clique), forming a new $(k+1)$ clique $C_{u}$ that is a superset of $P_{u}$. In $T(G)$, the addition of $u$ results in a new node $C_{u}$ being added and connected to $P_{u}$. Additionally, the $k$ many $k$-cliques in $C_{u}$ that contain the new vertex $u$ are added as new nodes to $T(G)$ and connected to $C_{u}$. From these observations it is clear that $T(G)$ is indeed a tree. $T(G)$.

We continue by proving some basic properties of our tree representation

Lemma 2. For any $k$-tree $G$ and any vertex $v \in V(G)$, the nodes of $T(G)$ that contain $v$ form a subtree of $T(G)$.

Proof. We prove by induction over the construction of $G$ that any node $M$ added to $T(G)$ with $v \in M$ either is the unique node first introducing $v$ or is hooked up to a previously added node that contains $v$. If $M$ is a $k$-clique in $G$ this is immediately clear as it is either the base node in $T(G)$ or it is a subset of a $(k+1)$-clique node and hence does not introduce any new vertices. So assume that $M$ is a $(k+1)$-clique $C_{u}$, which was added to $T(G)$ upon the addition of some vertex $u$ to $G$. If $u=v$ then $M$ is the single node of $T(G)$ introducing $v$. If $u \neq v$ we have $v \in C_{u} \backslash\{u\}=P_{u}$ and thus there is an edge to a previously added $k$-clique node $P_{u}$ that contains $v$.

Lemma 3. For any $k$-tree $G$, the center of $T(G)$ is a single node.

Proof. Suppose not. Then the center consists of two adjacent nodes, one a $k$ clique and one a $(k+1)$-clique. This leads to a contradiction because $k$-clique nodes have even eccentricity while that of $(k+1)$-clique nodes is odd: All leaves are $k$-clique nodes, and $k$-cliques and $(k+1)$-cliques alternate on every path.

Definition 4. The clique corresponding to the center node of $T(G)$ is called kernel of $G$ and denoted $\operatorname{ker}(G)$.

Note that $\operatorname{ker}(G)$ can be either a $k$-clique or a $(k+1)$-clique, depending on the structure of $G$. The concept of the kernel of a $k$-tree was introduced in [GSS02]. The definition there is slightly different but the equivalence can be easily verified.

We continue by recalling some basic facts concerning undirected trees. 
Fact 5. Given an undirected tree $T$ and two nodes $u, v \in V(T)$, the distance $d_{T}(u, v)$ can be computed in FL.

Proof. Think of $T$ as rooted in $u$ and all edges directed away from $u$. The direction of an edge $e$ can be determined in logspace by computing the lexicographically-first Euler tour starting at $u$ that visits each edge once per direction (cf. [AM04]). Then the unique path from $v$ to $u$ can be found by always choosing the unique incoming edge as next step. Only the current node and the number of steps have to be remembered. Upon reaching $u$, output the number of steps taken.

Fact 6. The center of an undirected tree $T$ can be computed in FL.

Proof. We first show that the eccentricity $\operatorname{ecc}_{T}(u)$ of each node $u \in V(T)$ is computable in logspace. This can be done by iterating over all $v \in V(T)$, each time calculating $d_{T}(u, v)$ (this is possible in logspace by Fact 5 ). Only the maximum distance to $u$ has to be remembered, the result being $\operatorname{ecc}_{T}(u)$.

Observe now that also the maximum eccentricity $e c c_{\max }$ of all nodes $u \in V(T)$ is computable in logspace by iterating over all $u \in V(T)$. Then compute again the eccentricity of all nodes $u$, this time outputting $u$ if $e c c_{T}(u)=e c c_{\max }$.

Our goal is to canonize $G$ by using Lindell's algorithm [Lin92] to canonize $T(G)$. To achieve this, we declare the kernel $K$ of $G$ as the root of $T(G)$. As a consequence, we can identify each $(k+1)$-clique $M \in V(T(G)) \backslash\{K\}$ with the unique vertex $v \in M$ that is not present in the $k$-clique $M^{\prime}$ that lies next to $M$ on the path from $K$ to $M$ in $T(G)$. For later use, we denote this vertex by $v(M)$ and for each $v \in V(G) \backslash K$, we use $M_{v}$ to denote the unique $(k+1)$-clique $M \in V(T(G)) \backslash\{K\}$ with $v(M)=v$.

It is clear that $T(G)$ does not provide complete structural information about $G$, since the vertices in the kernel $K$ are indistinguishable in $T(G)$ and further, only one out of the $k$ edges between each added vertex $u$ and its support can be recovered from $T(G)$. To add the missing information, we give individual colors to the kernel vertices and color the nodes of $T(G)$ as well. Since the kernel $K$ of a given $k$-tree $G$ can be determined in logspace, we can simplify the notation by assuming that $K$ consists of the vertices $1, \ldots, k^{\prime}$, where $k^{\prime}=\|K\| \in\{k, k+1\}$ equals the size of $K$.

Definition 7. Let $G$ be a $k$-tree with vertex set $V(G)=\{1, \ldots, n\}$ and kernel $K=\left\{1, \ldots, k^{\prime}\right\}$. For each vertex $v$ of $G$, we denote by

$$
\boldsymbol{l}_{\boldsymbol{G}}(\boldsymbol{v})=\min \left\{d_{T(G)}(K, M) \mid M \in V(T(G)), v \in M\right\}
$$

the level of $v$ in $G$. Further, for any permutation $\pi \in S_{k^{\prime}}$, let $\boldsymbol{T}(\boldsymbol{G}, \boldsymbol{\pi})$ denote the directed colored tree obtained from $T(G)$ by choosing $K$ as the root and coloring each node $M \in V(T(G))$ by the set $\boldsymbol{c}(\boldsymbol{M})=\{c(v) \mid v \in M\}$, where

$$
\boldsymbol{c}(\boldsymbol{v})= \begin{cases}\pi(v) & \text { if } v \in \operatorname{ker}(G), \\ l_{G}(v)+k^{\prime} & \text { otherwise }\end{cases}
$$


The definition of $T(G, \pi)$ is similar to the construction of the colored tree $T(G, B, \theta)$ in [ADK07]. The main advantage of our construction lies in the fact that $T(G, \pi)$ can be directly constructed from $G$ in logspace, whereas the tree representation used in [ADK07] (which in turn is related to the decomposition defined in [KCP82]) is defined as the reachable subgraph of a mangrove derived from $G$. This allows us to decide $s t$-reachability in the tree $T(G, \pi)$ in logspace, an essential step to achieve our upper bound.

Another advantage of our construction comes with the usage of the kernel $K$ as a canonical base. This makes it superfluous to cycle through all $k$-cliques of $G$ (as in [ADK07]), leaving only the permutations of the vertices within $K$ to enumerate.

Lemma 8. For a k-tree $G$ and a permutation $\pi$ on the kernel $K$ of $G, T(G, \pi)$ can be computed in $\mathrm{FL}$.

Proof. It is clear that the nodes and edges of $T(G)$ can be determined in logspace: First iterate over all subsets $M$ of $V(G)$ of size $k$ (this requires space $k \log n$ ) and output $M$ as a node if $M$ is a $k$-clique in $G$. Likewise, find and output all $(k+1)$-cliques $M$, each time adding edges to all $k+1$ many $k$-cliques contained in $M$. The (intermediate) result $T(G)$ cannot be stored due to space limitations, but it is possible to recompute it as needed (as long as only a constant number of operations is chained).

Next determine the kernel $K$ of $G$ (Fact 6) and think of all edges in $T(G)$ directed away from $K$. As described in Fact 5 , the direction can be determined in logspace. It remains to compute the color $c(M)$ of each node $M \in V(T(G))$.

For each $v \in M$ calculate $c(v)$ by examining the unique path from $M$ to $K$ in $T(G)$ (the path can be found by following the unique incoming edge at each node). Store the length $\ell$ of the path and the position $p_{v}$ where $v$ was last found (this can be done in parallel for all $v \in M$ ). If $p_{v}=\ell$ (i.e. $v \in K$ ), then add the number $c(v)=\pi(v)$ to the color $c(M)$ of $M$. If $p_{v}<\ell$, add the number $c(v)=\ell-p_{v}+\|K\|$ to $c(M)$. The latter is correct, because by Lemma 2 the nodes containing $v$ form a subtree of $T(G)$ and thus the node that is closest to $K$ and contains $v$ is on the path from $K$ to $M$.

We will need to compute a canonical labeling of $T(G, \pi)$. We observe the following generalization of the logspace tree canonization algorithm.

Lemma 9. Lindell's algorithm [Lin92] can be extended to colored trees and to output not only a canonical form, but also a canonical labeling. This modification preserves the logarithmic space bound.

Proof sketch. Colors can be handled by extending the tree isomorphism order defined in [Lin92] by using color $(s)<\operatorname{color}(t)$ as additional condition (where $s$ and $t$ are the roots of the trees to compare). The canonical labeling can be computed by using a counter $i$ initialized to 0 : Instead of printing (the first letter of) the canon of a node $v$, increment $i$ and print " $v \mapsto i$ ".

Next we show that the colored tree representations of isomorphic $k$-trees are also isomorphic, provided that the kernels are labeled accordingly. 
Lemma 10. Let $\phi \in S_{n}$ be an isomorphism between two $k$-trees $G$ and $H$ with $V(G)=V(H)=\{1, \ldots, n\}$ and $\operatorname{ker}(G)=\operatorname{ker}(H)=K$. Then $\phi$ (viewed as a mapping from $V(T(G))$ to $V(T(H)))$ is an isomorphism between $T\left(G, \pi_{1}\right)$ and $T\left(H, \pi_{2}\right)$, provided that $\pi_{1}(u)=\pi_{2}(\phi(u))$ for all $u \in K$.

Proof. It can be easily checked that any isomorphism between $G$ and $H$ is also an isomorphism between $T(G)$ and $T(H)$ that maps the kernel $K$ of $G$ to the kernel of $H$, which equals $K$ by assumption. In order to show that the color of a node $M \in V\left(T\left(G, \pi_{1}\right)\right)$ coincides with the color of $\phi(M) \in V\left(T\left(H, \pi_{2}\right)\right)$, we prove the stronger claim that $c(v)=c(\phi(v))$ for all $v \in V(G)$. For $v \in K$, we have $c(\phi(v))=\pi_{2}(\phi(v))=\pi_{1}(v)=c(v)$ by assumption. Since $\phi$ must preserve the level of the vertices, it follows further for $v \in V(G) \backslash K$ that

$$
c(\phi(v))=l_{H}(\phi(v))+\|K\|=l_{G}(v)+\|K\|=c(v) .
$$

This completes the proof of the lemma.

Conversely, the next lemma shows that from any isomorphic copy $T$ of $T\left(G, \pi_{1}\right)$ we can easily derive an isomorphic copy $G^{\prime}$ of $G$. Moreover, any isomorphism $\phi$ between $T(G, \pi)$ and $T$ can be efficiently converted into an isomorphism between $G$ and $G^{\prime}$.

Lemma 11. Let $G$ be a $k$-tree and let $\pi$ be a permutation on the kernel $K$ of $G$. Then from any colored tree $T$ that is isomorphic to $T(G, \pi)$, an isomorphic copy $G^{\prime}$ of $G$ can be computed in logspace. Further, it is possible to compute in logspace an isomorphism between $G$ and $G^{\prime}$ from any given isomorphism between $T(G, \pi)$ and $T$.

Proof sketch. Construct $G^{\prime}$ as follows. Let $V\left(G^{\prime}\right)=\{1, \ldots, n\}$, where $n$ is $k$ plus the number of $(k+1)$-clique nodes in $T$ (we call $m \in V(T)$ an $\boldsymbol{l}$-clique node, if $l=\|c(m)\|)$. This is correct due to the one-to-one correspondence between the vertices $v \in V(G) \backslash K$ and the $(k+1)$-clique nodes $M_{v} \in T(G, \pi) \backslash\{K\}$. Next determine the center node $z$ of $T$ (see Lemma 6 ) and make $\left\{1, \ldots, k^{\prime}\right\}$ a clique in $G^{\prime}$, where $k^{\prime}=\|c(z)\|$. Further, for any non-center $(k+1)$-clique node $m \in V(T) \backslash\{z\}$, let $v(m)$ denote the corresponding vertex in $V\left(G^{\prime}\right)$ (to make this mapping unique, let $v(m)$ preserve the order of $(k+1)$-clique nodes in $V(T)$ ). Based on the color $c(m)=\left\{c_{1}, \ldots, c_{k+1}\right\}$ of $m$ add the following edges to $E\left(G^{\prime}\right)$ : For each $c_{i} \leq k^{\prime}$ add an edge $\left\{c_{i}, v(m)\right\}$ and for each $c_{i}>k^{\prime}$ with $c_{i}<c_{\max }=\max \left\{c_{i} \mid c_{i} \in c(m)\right\}$ add an edge $\left\{v(m), v\left(m^{\prime}\right)\right\}$, where $m^{\prime}$ is the $\left(c_{i}-k^{\prime}\right)$-th node on the path from $z$ to $m$. This completes the construction of $G^{\prime}$.

Now let $\phi$ be an isomorphism from $T(G, \pi)$ to $T$. Construct an isomorphism $\phi^{\prime}$ from $G$ to $G^{\prime}$ as follows. For $v \in K$, let $\phi^{\prime}(v)=\pi(v)$, and for $v \notin K$, let $\phi^{\prime}(v)=v\left(\phi\left(M_{v}\right)\right)$. By induction on the level of $v$ in $G$, it can be proven that this is indeed an isomorphism. Both constructions can easily be seen to be in logspace.

Now we are ready to prove our main result. 
Theorem 12. Given a $k$-tree $G$ with vertex set $V(G)=\{1, \ldots, n\}$ and kernel $K=\left\{1, \ldots, k^{\prime}\right\}$, a canonical labeling $\psi_{G} \in S_{n}$ can be computed in $\mathrm{FL}$.

Proof. In order to compute $\psi_{G}$ we iterate over all permutations $\pi \in S_{k^{\prime}}$ and compute a canonical labeling $\psi_{T(G, \pi)}$ for the colored tree $T(G, \pi)$ using the algorithm from Lemma 9. Let $\pi_{1}$ be one of the permutations that give rise to the lexicographically smallest colored tree $\psi_{T\left(G, \pi_{1}\right)}\left(T\left(G, \pi_{1}\right)\right)$. By applying Lemma 11, we can reconstruct from this tree an isomorphic copy canon $(G)$ of $G$ together with an isomorphism $\psi_{G}$ between $G$ and canon $(G)$. By Lemmas 8 and 9 , it is clear that $\psi_{G}$ is computable in logspace.

It remains to show that the canonical labelings of any two isomorphic $k$ trees $G$ and $H$ map these graphs to the same canon $\psi_{G}(G)=\psi_{H}(H)$. To see this, let $\pi_{1}, \pi_{2} \in S_{k^{\prime}}$ be two permutations that give rise to the lexicographically smallest trees $\psi_{T\left(G, \pi_{1}\right)}\left(T\left(G, \pi_{1}\right)\right)$ and $\psi_{T\left(H, \pi_{2}\right)}\left(T\left(H, \pi_{2}\right)\right)$, respectively. Since by Lemma 10 for any tree $T\left(G, \pi_{1}\right)$ that can be derived from $G$ via some permutation $\pi_{1}$ there is an isomorphic tree $T\left(H, \pi_{2}\right)$ that can be derived from $H$ via some permutation $\pi_{2}$ (and vice versa), it follows that $\psi_{T\left(G, \pi_{1}\right)}\left(T\left(G, \pi_{1}\right)\right)$ and $\psi_{T\left(H, \pi_{2}\right)}\left(T\left(H, \pi_{2}\right)\right)$ are equal, implying that $\operatorname{canon}(G)=\operatorname{canon}(H)$.

We note that the above construction can be extended to colored $k$-trees as follows. Let $\zeta: V(G) \rightarrow C$ be a vertex coloring of $G$. Modify the coloring of $T(G, \pi)$ (cf. Definition 7) by replacing $c(v)$ with the pair $c^{\prime}(v)=(c(v), \zeta(v))$.

Theorem 12 immediately yields the following corollaries.

Corollary 13. For any fixed $k$, $k$-tree canonization is in $\mathrm{FL}$.

Corollary 14. For any fixed $k, k$-tree isomorphism is L-complete.

The L-hardness can be seen by a reduction from the isomorphism problem for trees in pointer notation, which is known to be L-hard $\left[\mathrm{JKM}^{+} 03\right]$. The reduction transforms a tree $T$ into a $k$-tree $\boldsymbol{E}_{\boldsymbol{k}}(\boldsymbol{T})$ by adding a $(k-1)$-clique $C$ and connecting $C$ to all nodes in $V(T)$ (cf. Fig. 2). It can easily be seen that $E_{k}(T)$ is a $k$-tree and that $T_{1} \cong T_{2} \Leftrightarrow E_{k}\left(T_{1}\right) \cong E_{k}\left(T_{2}\right)$.

We note that fixing $k$ is essential, as the isomorphism problem for the class of all $k$-trees, $k \in \mathbb{N}^{+}$, is isomorphism complete [KCP82] and thereby unlikely to be decidable in polynomial time.

Furthermore, there is a standard Turing reduction of the automorphism group problem (i.e., computing a generating set of the automorphism group of a given graph) to the search version of GI for colored graphs (cf. [Hof82,KST93]). It is not hard to see that this reduction can be performed in logspace.

Corollary 15. For any fixed $k$, computing a generating set of the automorphism group of a given $k$-tree, and hence computing a canonical labeling coset for a given $k$-tree is in $\mathrm{FL}$.

Corollary 16. For any fixed $k$, the $k$-tree automorphism problem (i. e., deciding whether a given $k$-tree has a non-trivial automorphism) is L-complete. 
Observe that the mapping $T \mapsto E_{k}(T)$ does not provide a correct reduction of tree automorphism (which is L-complete $\left[\mathrm{JKM}^{+} 03\right]$ ) to $k$-tree automorphism, as the vertices within the newly added clique can always be permuted without changing the graph. To sidestep this difficulty, we use a transformation $E_{k}^{\prime}$ that preserves rigidity. Let $T$ be a rooted tree with $n=\|V(T)\|$ and root $r \in V(T)$. Then $\boldsymbol{E}_{\boldsymbol{k}}^{\prime}(\boldsymbol{T}, \boldsymbol{r})$ is defined as follows (cf. Fig. 2):

$$
\begin{aligned}
V\left(E_{k}^{\prime}(T, r)\right)= & V(T) \cup\left\{u_{i} \mid 1 \leq i \leq k+n\right\} \\
E\left(E_{k}^{\prime}(T, r)\right)= & E(T) \cup\left\{\left\{v, u_{i}\right\} \mid v \in V(T), 1 \leq i \leq k-1\right\} \\
& \cup\left\{\left\{r, u_{k}\right\}\right\} \cup\left\{\left\{u_{i}, u_{j}\right\} \mid 1 \leq i<j \leq k+n, j-i \leq k\right\}
\end{aligned}
$$

It is easy to see that $E_{k}^{\prime}(T, r)$ is a $k$-tree and that any non-trivial automorphism of $(T, r)$ induces a non-trivial automorphism of $E_{k}^{\prime}(T, r)$. To see that $E_{k}^{\prime}(T, r)$ is rigid whenever $(T, r)$ is rigid, assume $n>k$ (all smaller trees can be hardcoded in the reduction). Any automorphism of $E_{k}^{\prime}(T, r)$ must fix all newly added vertices $u_{i}$ : Each of the vertices $u_{i}, 1 \leq i \leq k-1$, is uniquely determined by its degree $n+k-2+i$ (unless $r$ is connected to all vertices of $T$, but then $T$ is a star and not rigid anyway). The vertices $u_{i}, k+1 \leq i \leq k+n$, are the only ones not adjacent to $u_{1}$ and uniquely identified by the structure of $E_{k}^{\prime}(T, r)$ (examine the tree representation $T\left(E_{k}^{\prime}(T, r)\right)$ to see this). Finally, the vertex $u_{k}$ is unique among the remaining ones by the shortest distance to $u_{k+n}$.
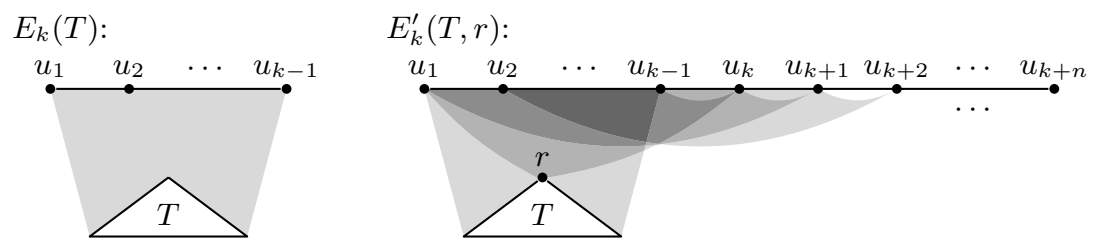

Fig. 2. The transformations $E_{k}(T)$ and $E_{k}^{\prime}(T, r)$

\section{Complete problems for logspace}

In this section we prove some additional completeness results for logspace that are related to our main result. The hardness is under DLOGTIME-uniform $A C^{0}$ reductions. We first recall that ORD is L-complete, where ORD is the problem of deciding for a directed line graph $P$ and two vertices $s, t \in V(P)$ if there is a path from $s$ to $t$ [Ete97].

In Lemma 6 we have seen that the center of an undirected tree can be computed in FL. We now show that the decision variant is hard for $L$ even when restricted to paths.

Theorem 17. Given an undirected path $P$ and a vertex $c \in V(P)$, it is L-hard to decide if $c$ belongs to the center of $P$. 
This implies the L-hardness of the following problem: Given a $k$-tree (or $k$-path) $G$ and a vertex $c \in V(G)$, decide whether $c$ belongs to the kernel of $G$. The reduction for this is $(P, c) \mapsto\left(E_{k}(P), c\right)$, where $E_{k}$ is as defined above.

Proof. We reduce from ORD using $(P, s, t) \mapsto\left(P^{\prime}, n\right)$ as reduction, where

$$
\begin{aligned}
V\left(P^{\prime}\right)= & V(P) \cup\left\{i^{\prime} \mid i \in V(P)\right\} \cup\left\{s^{\prime \prime}\right\} \\
E\left(P^{\prime}\right)= & \{\{i, j\} \mid(i, j) \in E(P) \wedge j \neq t\} \cup\left\{\left\{n, n^{\prime}\right\}\right\} \\
& \cup\left\{\left\{i^{\prime}, j^{\prime}\right\} \mid(i, j) \in E(P) \wedge j \notin\{s, t\}\right\} \\
& \cup\left\{\left\{i^{\prime}, s^{\prime \prime}\right\} \mid(i, s) \in E(P)\right\} \cup\left\{\left\{s^{\prime \prime}, s^{\prime}\right\}\right\}
\end{aligned}
$$

and $n$ is the vertex without successor in $P . P^{\prime}$ is the undirected path that consists of two copies of $P$ that are twisted before $t$, connected at their ends and have the second copy of $s$ duplicated (cf. Fig. 3). If $s$ precedes $t$ in $P$ (left side), then $n$ is the center of $P^{\prime}$, but if $t$ precedes $s$ then $n^{\prime}$ is the center of $P^{\prime}$ (right side).

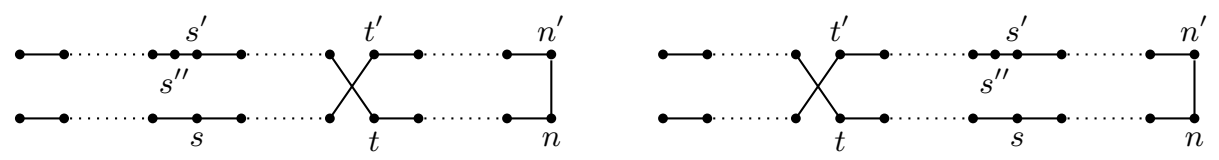

Fig. 3. The reduction of ORD to verifying the center

Finally, we examine two problems related to the structure of $k$-trees. Let $G$ be a graph. A vertex $v \in V(G)$ is called simplicial in $G$, if its neighborhood induces a clique. A bijective mapping $\sigma:\{1, \ldots,\|V(G)\|\} \rightarrow V(G)$ of the vertices of $G$ is called perfect elimination order (PEO), if for all $i, \sigma(i)$ is simplicial in $G-\bigcup_{j<i}\{\sigma(j)\}$. Note that a graph can have several perfect elimination orders, so finding a $\mathrm{PEO}$ is not a functional but a search problem. It is well-known that a graph has a PEO if and only if it is chordal. As $k$-trees are a subclass of chordal graphs, each $k$-tree has a PEO.

A related problem is the fast reordering problem (FRP) which is defined in [GSS02] as a preprocessing step for parallel algorithms. It consists of finding a sequence of sets $R_{0}, \ldots, R_{k} \subseteq V(G)$, such that each $R_{i}$ is a maximal independent set of simplicial vertices of $G-\bigcup_{j<i} R_{j}$ and that $G-\bigcup_{0 \leq j \leq k} R_{j}$ is a clique. For general chordal graphs there can be several such sequences, but for $k$-trees this sequence is unique and the remaining clique is the kernel. In [GSS02] it was shown that if the input graphs are restricted to $k$-trees, the FRP can be solved in NC. We improve this and show logspace completeness for both problems:

Theorem 18. For $k$-trees ( $k$ fixed), it is logspace complete to find a perfect elimination order and to solve the fast reordering problem.

Proof. We first show FRP $\in$ FL: Let $G$ be a $k$-tree. We compute the level $l_{G}(v)$ for each $v \in V(G)$ (cf. Definition 7). As observed in Lemma 8, this is possible in 
logspace. Let $l_{\max }=\max \left\{l_{G}(v) \mid v \in V(G)\right\}$. Output $R_{i}:=\left\{v \in V(G) \mid l_{G}(v)=\right.$ $\left.l_{\max }-2 i\right\}$ for $i=0, \ldots,\left\lceil l_{\max } / 2\right\rceil-1$. The correctness follows from the structure of $T(G)$.

Next, we note that a perfect elimination order can be efficiently computed when a solution to the FRP is known (i. e. finding a PEO reduces to solving the FRP): Take the members of the $R_{i}$ in ascending order (first those from $R_{0}$, then those from $R_{1}$ and so on up to $R_{k}$ ) and finally those from $\operatorname{ker}(G)=V(G) \backslash \bigcup_{i} R_{i}$. No matter which order is chosen within the $R_{i}$ and the kernel, the result is a $\mathrm{PEO}$, as each $R_{i}$ is independent and $\operatorname{ker}(G)$ is a clique.

Finally we show that finding a perfect elimination order is hard for logspace even for paths. The result for $k$-trees (and $k$-paths) can again be obtained using the construction of $E_{k}$ given above. We solve an ORD instance $(P, s, t)$ in DLOGTIME-uniform $\mathrm{AC}^{0}$ with a single oracle gate for computing a PEO of the path $P^{\prime}$ given by

$$
\begin{aligned}
V\left(P^{\prime}\right)= & V(P) \cup\left\{i^{\prime} \mid i \in V(P) \backslash\{n\}\right\} \\
E\left(P^{\prime}\right)= & \{\{i, j\} \mid(i, j) \in E(P)\} \\
& \cup\left\{\left\{i^{\prime}, j^{\prime}\right\} \mid(i, j) \in E(P), j \neq n\right\} \cup\left\{\left\{i^{\prime}, n\right\} \mid(i, n) \in E(P)\right\}
\end{aligned}
$$

where $n$ is the vertex in $P$ without successor. We claim that for any PEO $\sigma$ of $P^{\prime}$ (where $p_{i}$ is a shorthand for the position $\sigma^{-1}(i)$ of a vertex in $\sigma$ ):

$$
(P, s, t) \in \mathrm{ORD} \Leftrightarrow p_{s} \leq p_{t} \leq p_{n} \vee p_{s^{\prime}} \leq p_{t^{\prime}} \leq p_{n}
$$

If $(P, s, t) \notin \mathrm{ORD}$, then $s$ is between $t$ and $n$, and $s^{\prime}$ is between $t^{\prime}$ and $n$ in $P^{\prime}$ (right side in Fig. 4). Thus $\sigma$ cannot satisfy both $p_{s} \leq p_{t}$ and $p_{s^{\prime}} \leq p_{t^{\prime}}$. If $(P, s, t) \in$ ORD (left side of Fig. 4), $n$ does not become simplicial until at least one copy of $P$ is completely removed. Similarly, if the first copy is completely removed before $n, t$ does not become simplicial before $s$ is removed; and if the second copy is completely removed before $n, t^{\prime}$ does not become simplicial before $s^{\prime}$ is removed.
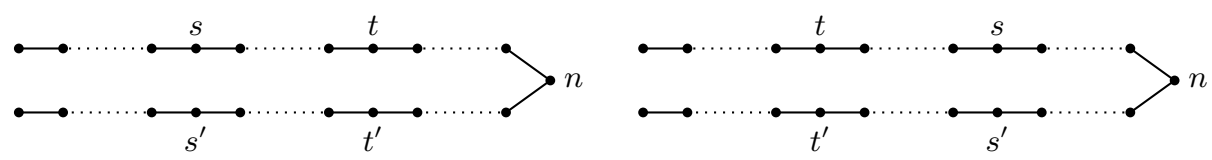

Fig. 4. The reduction of ORD to finding a PEO

\section{References}

[ADK07] Arvind, V., Das, B., Köbler, J.: The space complexity of $k$-tree isomorphism. In: Algorithms and Computation. Proceedings of 18th ISAAC. LNCS 4853, Springer (2007) 822-833 
[ADK08] Arvind, V., Das, B., Köbler, J.: A logspace algorithm for partial 2-tree canonization. In: Proceedings of the 3rd International Computer Science Symposium in Russia (CSR). LNCS 5010, Springer (2008) 40-51

[AHU74] Aho, A., Hopcroft, J., Ullman, J.: The design and analysis of computer algorithms. Addison-Wesley (1974)

[AJ93] Àlvarez, C., Jenner, B.: A very hard log-space counting class. Theoretical Computer Science 107(1) (1993) 3-30

[AK06] Arvind, V., Kurur, P.P.: Graph isomorphism is in SPP. Information and Computation 204(5) (2006) 835-852

[AM04] Allender, E., Mahajan, M.: The complexity of planarity testing. Information and Computation 139(1) (February 2004)

$\left[\mathrm{BDH}^{+}\right.$92] Buntrock, G., Damm, C., Hertrampf, U., Meinel, C.: Structure and importance of logspace-MOD classes. Mathematical Systems Theory 25 (1992) 223-237

[Coo85] Cook, S.A.: A taxonomy of problems with fast parallel algorithms. Information and Control 64 (1985) 2-22

$\left[\mathrm{DLN}^{+} 08\right]$ Datta, S., Limaye, N., Nimbhorkar, P., Thierauf, T., Wagner, F.: A log-space algorithm for canonization of planar graphs. CoRR (2008) http://arxiv.org/abs/0809.2319

[Ete97] Etessami, K.: Counting quantifiers, successor relations, and logarithmic space. Journal of Computer and System Sciences 54(3) (1997) 400-411

[GS86] Goldwasser, S., Sipser, M.: Private coins versus public coins in interactive proof systems. In: Randomness and Computation. Volume 5 of Advances in Computing Research. JAI Press (1989) 73-90

[GSS02] Del Greco, J.G., Sekharan, C.N., Sridhar, R.: Fast parallel reordering and isomorphism testing of $k$-trees. Algorithmica 32(1) (2002) 61-72

[Hof82] Hoffmann, C.: Group-Theoretic Algorithms and Graph Isomorphism. LNCS 136. Springer (1982)

$\left[\mathrm{JKM}^{+}\right.$03] Jenner, B., Köbler, J., McKenzie, P., Torán, J.: Completeness results for graph isomorphism. Journal of Computer and System Sciences 66 (2003) $549-566$

[KCP82] Klawe, M.M., Corneil, D.G., Proskurowski, A.: Isomorphism testing in hookup classes. SIAM Journal on Algebraic and Discrete Methods 3(2) (June 1982) 260-274

[KST93] Köbler, J., Schöning, U., Torán, J.: The Graph Isomorphism Problem: Its Structural Complexity. Progress in Theoretical Computer Science. Birkhäuser, Boston (1993)

[Lin92] Lindell, S.: A logspace algorithm for tree canonization. extended abstract. In: Proceedings of the 24th STOC, New York, ACM (1992) 400-404

[MR91] Miller, G., Reif, J.: Parallel tree contraction part 2: further applications. SIAM Journal on Computing 20 (1991) 1128-1147

[Rei05] Reingold, O.: Undirected st-connectivity in log-space. In: Proceedings of the 37th STOC, New York, ACM (2005) 376-385

[Sch88] Schöning, U.: Graph isomorphism is in the low hierarchy. Journal of Computer and System Sciences 37 (1988) 312-323

[Tor04] Torán, J.: On the hardness of graph isomorphism. SIAM Journal on Computing 33(5) (2004) 1093-1108 